\title{
ESTUDO CINÉTICO DA REMOÇÃO DE MATÉRIA ORGÂNICA DE ÁGUAS RESIDUÁRIAS DA SUINOCULTURA EM SISTEMAS ALAGADOS CONSTRUÍDOS CULTIVADOS COM DIFERENTES ESPÉCIES DE VEGETAIS
}

\author{
ANTONIO T. DE MATOS ${ }^{1}$, WALLISSON DA S. FREITAS ${ }^{2}$, ALISSON C. BORGES ${ }^{3}$
}

RESUMO: Com a realização deste estudo, teve-se o objetivo de obter valores dos coeficientes de remoção da matéria orgânica de águas residuárias da suinocultura (ARS), quando em tratamento em sistemas alagados, construídos (SACs) e cultivados com diferentes espécies vegetais e em condições climáticas tropicais. Em tanques de 24,0 x 1,1 x 0,7 m, impermeabilizados com lona de PVC e preenchidos com uma camada de $0,4 \mathrm{~m}$ de brita zero (altura útil), foram plantados taboa (SAC1), tripa-de-sapo (SAC2) e capim-tifton 85 (SAC3). No SAC4 (multivegetado), foi plantado, no primeiro terço, tripa-de-sapo; no segundo terço, taboa, e no último terço, capim-tifton 85 . A ARS foi previamente tratada em filtros orgânicos constituídos por bagaço de cana-de-açúcar, sendo aplicada numa vazão de $0,8 \mathrm{~m}^{3} \mathrm{~d}^{-1}$, o que correspondeu a um tempo de retenção hidráulica (TRH) aproximado de 6,4 dias. Coletaram-se amostras da água residuária nos pontos 0 (entrada), 4; $8 ; 12$; 16; 20 e 24 m (saída) de cada SAC. Nas amostras coletadas, foram analisadas as concentrações de DQO, sendo os valores utilizados para a obtenção dos parâmetros cinéticos aparentes. Não houve diferença $(\mathrm{p}>0,05)$ entre os SACs, na capacidade do sistema em remover DQO; entretanto, a época do ano teve influência na dinâmica da remoção de matéria orgânica nos sistemas. Os valores do coeficiente cinético aparente $\mathrm{k}_{\mathrm{v}}$ ' ficaram entre 0,94 e $1,52 \mathrm{~d}^{-1}$ no cultivo, no período de verão. Já no período de outono/inverno, o coeficiente $\mathrm{k}_{\mathrm{v}}{ }^{\prime}$ apresentou valores menores, entre 0,88 e 1,07 $\mathrm{d}^{-1}$.

PALAVRAS-CHAVE: efluentes suinícolas, zona de raízes, cinética de remoção, fitorremediação.

\section{KINETIC STUDY OF ORGANIC MATTER REMOVAL FROM SWINE WASTEWATERS TREATED IN CONSTRUCTED WETLANDS CULTIVATED WITH DIFFERENT SPECIES OF PLANTS}

\begin{abstract}
The objective of this study was obtain the coefficients of organic matter removal in constructed wetlands (CWs) cultivated with different plant species, in tropical climatic conditions, when used in swine wastewater treatment. In tanks of $24.0 \mathrm{~m} \times 1.1 \mathrm{~m} \times 0.7 \mathrm{~m}$, sealed with PVC canvas, filled with a layer $(0.4 \mathrm{~m}$ of depth) of fine gravel were planted cattail $(\mathrm{CW} 1)$, alligator weed (CW2) and tifton 85 bermudagrass (CW3). In CW4, was planted, on the $1^{\text {st }}$ third, alligator weed; on the $2^{\text {nd }}$ third, cattail and in the last third, tifton 85 bermudagrass. The swine wastewater, previously treated by organic filters made of bagasse from sugar cane, was applied at rate of $0.8 \mathrm{~m}^{3} \mathrm{~d}^{-1} \mathrm{in} \mathrm{CWs}$, which corresponded to a hydraulic retention time (HRT) of 6.4 days, approximately. Wastewater samples were collected in sections 0 (entry), 4; 8; 12; 16; 20 and 24 m (output) of each CW. In the samples collected were analyzed the concentrations of COD and the obtained values were used to adjust apparent kinetic parameters. There was no difference ( $p>0.05)$ among the CWs, in respect the COD removal, however the year season influenced the dynamics of organic matter removal in CWs performance. The values of the parameter $\mathrm{k}_{\mathrm{v}}$ ' were, during the summer, between 0.94 and $1.52 \mathrm{~d}^{-1}$. During the autumn/winter season, the $\mathrm{k}_{\mathrm{v}}$ ' coefficient showed lower values, between 0.88 and $1.07 \mathrm{~d}^{-1}$.
\end{abstract}

KEYWORDS: swine wastewater, root zones, removal kinetics, phytoremediation.

\footnotetext{
${ }^{1}$ Eng ${ }^{\circ}$ Agrícola, Dr. em Solos e Nutrição de Plantas, Prof. Associado do Departamento de Engenharia Agrícola, UFV, Viçosa - MG.

${ }^{2}$ Eng $^{\mathrm{O}}$ Agrícola, Prof. Doutor, Instituto Federal de Educação, Ciência e Tecnologia do Norte de Minas Gerais, Campus Januária, IFNMG, Januária, MG. Email: wallissonfreitas@yahoo.com.br.

${ }^{3}$ Eng. Civil, Dr. em Eng. Hidráulica e Saneamento, Prof. Adjunto do Departamento de Engenharia Agrícola, UFV, Viçosa - MG, Fone: (0XX31) 3899.1876, borges@ufv.br . 


\section{INTRODUÇÃO}

Dentre as propostas para tratamento de águas residuárias ricas em material orgânico, como é o caso dos efluentes provenientes de granjas suinícolas, distingue-se a utilização de sistemas alagados construídos (SACs), também denominados constructed wetlands. Tais sistemas configuram-se como alternativas simplificadas, apresentando reduzidos custos de implantação e operação para o tratamento desses resíduos.

As plantas, o substrato e o biofilme de microrganismos que se formam no meio são os responsáveis, direta ou indiretamente, pela remoção de poluentes da água residuária sendo, por essa razão, componentes fundamentais de um SAC. Os microrganismos desenvolvem-se aderidos às superfícies do material do meio suporte, do caule e da raiz de plantas e nos sólidos suspensos acumulados no sistema. A aeração do meio é feita via rizomas das plantas e por difusão do ar atmosférico na superfície do meio suporte, possibilitando a formação de sítios aeróbios e anaeróbios dentro do canal, característicos nesses sistemas (KADLEC \& WALLACE, 2009; VYMAZAL \& KRÖPFELOVÁ, 2009).

Os SACs podem ser cultivados em monocultura, com apenas uma espécie vegetal, ou serem cultivados com uma seleção de espécies (TROTTER et al., 1994). A escolha da espécie em SACs é de fundamental importância para o sucesso do tratamento, juntamente com outras variáveis de dimensionamento. Dentre as funções da parte vegetal do sistema, estão incluídas: remoção de nutrientes da água residuária; transferência de oxigênio para o substrato; servir de suporte (rizomas e raízes) para o crescimento de biofilme de microrganismos, além de melhoria na permeabilidade do substrato e na estética do ambiente. Nos EUA, as principais espécies utilizadas no tratamento de águas residuárias têm sido o junco de lagoa (Scirpus sp.), a taboa (Typha sp.) e o junco-manso (Eleocharis sp.). No Brasil, algumas avaliações têm sido feitas com o cultivo da taboa (BORGES et al., 2009), do capim tifton 85 (Cynodon spp.) (MATOS et al., 2008), da aveia-preta (Avena strigosa) (FIA et al., 2010) e do capim-elefante cv. Napier (Pennisetum purpureum) (MATOS et al., 2010a; MATOS et al., 2010b).

Modelos baseados na cinética de primeira ordem, aplicáveis a reatores tipo pistão, são os mais amplamente utilizados para se calcular o volume e a área superficial necessários para se efetuar o tratamento secundário de águas residuárias, e estes modelos têm sido utilizados em SACs (REED et al., 1995; VYMAZAL \& KRÖPFELOVÁ, 2009), sendo a constante $\mathrm{k}_{\mathrm{v}}$ obtida com o ajuste da eq.(1):

$$
\mathrm{C} / \mathrm{C}_{\mathrm{a}}=\mathrm{e}^{\left(-\mathrm{k}_{\mathrm{v}} \text { TRH }\right)}
$$

em que,

$\mathrm{C}_{\mathrm{a}}$ - concentração do poluente no afluente, $\mathrm{g} \mathrm{m}^{-3}$;

$\mathrm{C}$ - concentração do poluente no líquido residente, $\mathrm{g} \mathrm{m}^{-3}$;

$\mathrm{k}_{\mathrm{V}}$ - constante de reação de primeira ordem, base volumétrica, $\mathrm{d}^{-1}$, e

TRH - tempo de retenção hidráulica, d.

BRASIL et al. (2007), em estudos em SACs cultivados com taboa, no tratamento de esgoto doméstico, verificaram que o modelo de remoção de $1^{a}$ ordem de matéria orgânica não se ajustou bem aos dados observados, uma vez que, segundo os autores, os valores de $\mathrm{k}_{\mathrm{v}}$ deveriam ser alterados ao longo do comprimento dos SACs, dado o aumento na resistência do material orgânico que permanece para ser degradado nas posições mais à jusante do tanque. Os autores propuseram, então, uma modificação no modelo matemático, tal como apresentado na eq.(2), e obtiveram, com boas correlações, valores de $\mathrm{k}_{\mathrm{v}}$ ' iguais a 1,62 e expoente $\mathrm{n}$ igual a 0,25 , para corrigir a menor degradabilidade do material orgânico resiliente nos SACs.

$$
\mathrm{C} / \mathrm{C}_{\mathrm{a}}=\mathrm{e}^{\left(-\mathrm{k}_{\mathrm{v}}{ }^{\prime}{ }^{T R} \mathrm{H}^{\mathrm{n}}\right)}
$$


em que,

$\mathrm{k}_{\mathrm{v}}{ }^{\prime}$ - constante de reação de primeira ordem, base volumétrica, $\mathrm{d}^{-1}, \mathrm{e}$

n - coeficiente de aumento da resistência à degradação do material orgânico e redução do tempo (adimensional).

HUNT \& POACH (2001) compilaram dados promissores de uso de SACs no Hemisfério Norte para o tratamento de águas residuárias agroindustriais, como efluentes de suinocultura. No entanto, ainda é insuficiente o conhecimento sobre aspectos como a influência da espécie cultivada no SAC e o efeito das condições climáticas (época do ano) no desempenho dos sistemas. Dessa maneira, tornam-se necessários estudos de monitoramento e avaliação desses sistemas, de forma a se disponibilizar coeficientes cinéticos de remoção, mais apropriados para utilização em SACs a serem operados sob condições de clima tropical.

Com a realização deste estudo, objetivou-se obter valores das constantes de reação de primeira ordem da equação proposta por BRASIL et al. (2007), em SACs cultivados com diferentes espécies vegetais e operados em duas épocas do ano.

\section{MATERIAL E MÉTODOS}

O experimento foi conduzido na Área Experimental de Hidráulica, Irrigação e Drenagem do Departamento de Engenharia Agrícola da Universidade Federal de Viçosa - DEA/UFV, em Viçosa, Minas Gerais.

A ARS usada no experimento foi captada, por bombeamento, de um tanque de alvenaria de dimensões de $4 \times 5 \times 2 \mathrm{~m}$, utilizado para coletar as águas residuárias provenientes da Suinocultura da UFV, localizada próxima à referida área experimental. Antes de bombear a ARS para a Estação Experimental de Tratamento, fazia-se, por meio de sistemas de registros e tubos, a recirculação da ARS, a fim de homogeneizá-la no tanque. Na Figura 1, está apresentado um esquema das unidades de experimentação.

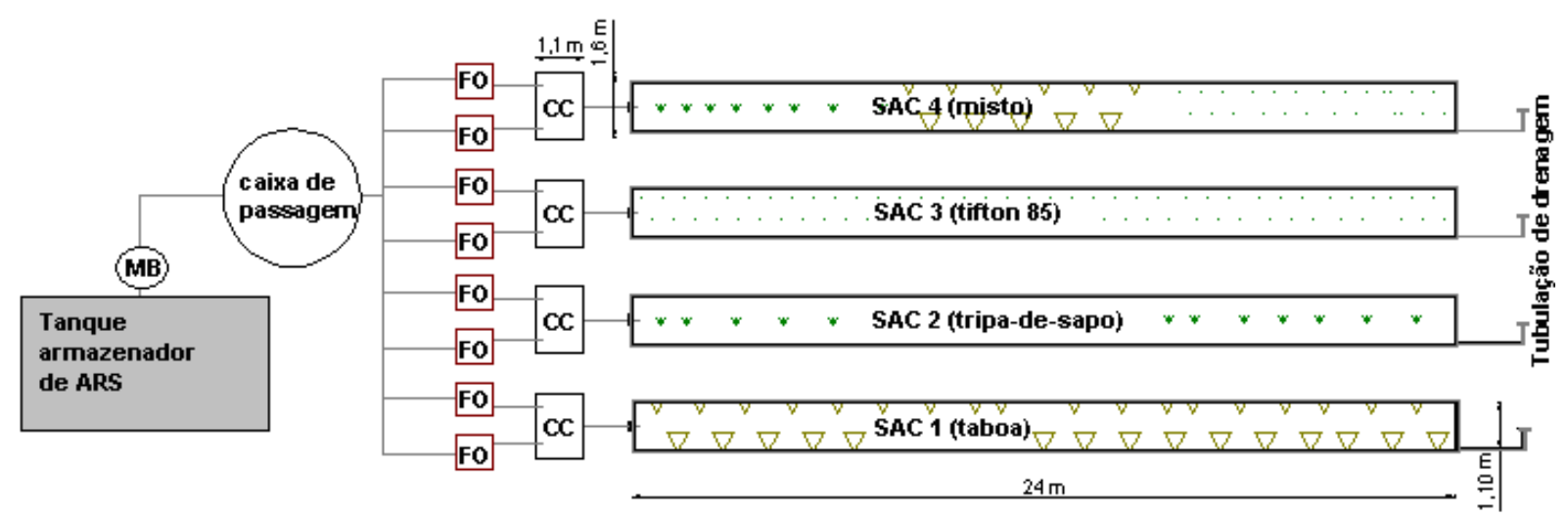

MB: conjunto motobomba; FO: filtro orgânico (bagaço de cana-de-açúcar); CC: caixa de controle da vazão aplicada.

FIGURA 1. Perfil esquemático da Estação Experimental de Tratamento. Scheme of Experimental Station.

O experimento foi constituído por quatro sistemas alagados construídos com escoamento subsuperficial horizontal (SACs) para tratamento de ARS, construídos paralelamente em valas escavadas no solo, com dimensões de $0,7 \mathrm{~m}$ de altura (sendo $0,4 \mathrm{~m}$ de substrato), 1,1 $\mathrm{m}$ de largura e 24,0 m de comprimento, impermeabilizados com geomembrana de policloreto de vinila (PVC), com espessura de $0,5 \mathrm{~mm}$. Todos os SACs tinham declividade do fundo de $0,005 \mathrm{~m} \mathrm{~m}^{-1}$. O nível da água era mantido rente ao nível do substrato no final dos SACs. Como meio de suporte, utilizou-se brita zero (diâmetro $\mathrm{D}_{60}$ igual a $7,0 \mathrm{~mm}$, coeficiente de uniformidade $\mathrm{D}_{60} / \mathrm{D}_{10}$ igual a 1,6 , volume de vazios de $0,49 \mathrm{~m}^{3} \mathrm{~m}^{-3}$ e condutividade hidráulica do meio saturada de $7.970 \mathrm{~m} \mathrm{~d}^{-1}$ ). 
A ARS bombeada até a área experimental era, a cada aplicação, conduzida para uma caixa de passagem com capacidade de $5 \mathrm{~m}^{3}$ a fim de facilitar o manejo e a distribuição nos SACs. A fim de proceder a um tratamento primário, a água residuária, antes de ser aplicada nos SACs, passou por filtro orgânico com leito de bagaço de cana-de-açúcar picado, seguindo-se recomendações de MAGALHÃES et al. (2006).

A ARS filtrada era, então, conduzida para caixas de controle com capacidade de $1 \mathrm{~m}^{3}$, graduadas e providas de válvulas esféricas, por onde se podia controlar a vazão a ser aplicada diretamente nos SACs. O afluente aplicado nos SACs apresentou matéria orgânica, macro e micro-nutrientes nas seguintes concentrações médias, considerando-se 11 amostragens realizadas ao longo do período experimental: DQO de $1.950 \mathrm{~g} \mathrm{~m}^{-3}$; DBO de $511 \mathrm{~g} \mathrm{~m}^{-3}$; nitrogênio total equivalente a $308 \mathrm{~g} \mathrm{~m}^{-3}$; fósforo total igual a $73 \mathrm{~g} \mathrm{~m}^{-3}$; potássio igual a $120 \mathrm{~g} \mathrm{~m}^{-3}$ e concentração de zinco equivalente a $5 \mathrm{~g} \mathrm{~m}^{-3}$.

Em três dos SACs cultivados, foi plantada uma única espécie vegetal (taboa, tripa-de-sapo ou capim tifton 85), em outro foram plantadas, em seqüência, as três espécies vegetais em análise; assim, a distribuição dos tratamentos foi feita da seguinte forma:

- SAC1: totalmente cultivado com taboa (Typha latifolia $\mathrm{L}$. );

- SAC2: totalmente cultivado com tripa-de-sapo (Alternanthera philoxeroides (Mart) Griseb);

- SAC3: totalmente cultivado com capim tifton 85 (Cynodon dactylon (L.) Pers.);

- SAC4: cultivado no primeiro terço com tripa-de-sapo, no segundo terço com taboa e no terço final com tifton 85.

O plantio de todas as espécies vegetais foi iniciado entre os meses de abril e maio de 2004, e a aplicação diária da ARS teve início em dezembro do mesmo ano. Em cada SAC, foram aplicados $0,8 \mathrm{~m}^{3}$ de ARS filtrada por dia, o que correspondeu a uma taxa de aplicação média de $154,8 \mathrm{~kg}$ DBO ha ${ }^{-1} \mathrm{~d}^{-1}$ (equivalente a 590,9 $\mathrm{kg}$ DQO ha ${ }^{-1} \mathrm{~d}^{-1}$ ), distribuindo-se a aplicação continuamente ao longo do dia, correspondendo a um TRH aproximado de 6,4 dias.

A coleta de amostras da água residuária em tratamento foi feita por meio de tubos de PVC com $32 \mathrm{~mm}$ de diâmetro, inseridos verticalmente no material suporte, até atingir a geomembrana impermeável, posicionados a $0 ; 4 ; 8 ; 12 ; 16 ; 20$ e $24 \mathrm{~m}$, no sentido longitudinal dos SACs. Na manhã anterior ao dia de coleta das amostras, os cinco pontos de coleta intermediários (tubos de amostragem) dos SACs eram drenados, utilizando-se de bombas de sucção, para evitar a coleta de resíduos de lodos acumulados ou de água estagnada. Na manhã seguinte, coletavam-se as amostras com o uso de seringas feitas com tubos e conexões de PVC e mangueira flexível, succionando-se, em cada ponto de coleta, líquido do fundo, do meio e próximo à superfície. As amostragens do líquido em escoamento foram feitas entre fevereiro e setembro de 2005 , tendo sido, neste período, efetuadas 3 coletas no verão e 8 coletas no período de outono/inverno.

As análises de DQO foram feitas nas amostras coletadas nos, já citados, sete pontos de cada SAC. As amostras em cada ponto foram compostas por duas subamostras colhidas nos dois tubos, metade $\left(250 \mathrm{~cm}^{3}\right)$ em um tubo e o restante em outro, posicionado ao lado, sendo efetuadas no horário de 8 às 11 horas, sempre seguindo o sentido do início para o final dos SACs.

Durante as coletas, as amostras foram acondicionadas em caixas térmicas e, em seguida, conduzidas ao laboratório, para análise. A análise de DQO foi feita pelo método de oxidação química em refluxo aberto, no Laboratório de Qualidade da Água do Departamento de Engenharia Agrícola da UFV, em conformidade com recomendações do Standard Methods for the Examination of Water and Wastewater (APHA/AWWA/WEF, 2005). Os dados de temperatura foram obtidos na Estação Climatológica do DEA/UFV.

Como análise quantitativa dos dados, foram ajustadas, por regressão, equações matemáticas, utilizando-se do modelo de cinética de $1^{\mathrm{a}}$ ordem, modificado por BRASIL et al. (2007), tendo como função do tempo de retenção hidráulica, obtido a partir do valor de distância de percurso da água residuária nos SACs. Para tanto, foi utilizado o aplicativo Microcal Origin 8.0. Como análise 
estatística qualitativa dos dados, fez-se análise de variância para se verificar o efeito da espécie vegetal e da época de cultivo, nos valores de $\mathrm{k}_{\mathrm{v}}$ ' e $\mathrm{n}$, ajustados para as equações. Considerando-se os altos coeficientes de variação dos dados, comuns neste tipo de experimento, foram adotados níveis de significância mais elevados (até 15\%).

\section{RESULTADOS E DISCUSSÃO}

Na Tabela 1, estão apresentados os valores de eficiência de remoção de matéria orgânica registrados. Ressalta-se que, no período de verão, a temperatura média do ar foi sempre superior a $20{ }^{\circ} \mathrm{C}$. Já no período outono/inverno, a temperatura média observada na semana anterior às amostragens sempre foi $\leq 20{ }^{\circ} \mathrm{C}$.

TABELA 1. Valores (média \pm desvio) de eficiência (\%) de remoção de matéria orgânica (DQO) nos SACs tratando ARS filtrada. Efficiency (\%) values (average \pm deviation) of organic matter removal (COD) in the CWs treating filtered swine wastewater.

\begin{tabular}{lccccc}
\hline Período & $\begin{array}{c}\text { SAC1 } \\
\text { taboa }\end{array}$ & $\begin{array}{c}\text { SAC2 } \\
\text { tripa-de-sapo }\end{array}$ & $\begin{array}{c}\text { SAC3 } \\
\text { tifton } 85\end{array}$ & $\begin{array}{c}\text { SAC4 } \\
\text { misto }\end{array}$ & $\begin{array}{c}\text { Eficiência média } \\
\text { todos os SACs }\end{array}$ \\
\hline Verão & $87 \pm 10$ & $94 \pm 3$ & $93 \pm 4$ & $92 \pm 6$ & $92 \pm 6$ \\
Outono/Inverno & $88 \pm 9$ & $85 \pm 11$ & $89 \pm 10$ & $92 \pm 4$ & $88 \pm 9$ \\
Geral & $88 \pm 9$ & $87 \pm 10$ & $90 \pm 9$ & $92 \pm 4$ & $89 \pm 8$ \\
\hline
\end{tabular}

Os SACs apresentaram elevados valores de remoção de matéria orgânica, operando com um TRH equivalente a 6,4 d. Ressalta-se que todos os sistemas apresentaram eficiências superiores ao mínimo preconizado pela legislação estadual mineira, que equivale a uma eficiência média anual de $75 \%$ da remoção de DQO (COPAM/CERH, 2008).

Na Tabela 2, estão apresentados os valores médios de concentração de matéria orgânica (DQO) nos efluentes dos SACs, nos períodos de verão e outono/inverno. Foi observada diferença significativa $(\mathrm{p}<0,15)$ entre os valores de concentrações efluentes nos períodos de verão (temperaturas médias do ar no período variando entre 19,0 e $25,0{ }^{\circ} \mathrm{C}$, com média geral de $22,4{ }^{\circ} \mathrm{C}$ ) e outono/inverno (temperaturas médias do ar no período oscilando entre 10,8 e $21,9{ }^{\circ} \mathrm{C}$, com média geral de $17,4{ }^{\circ} \mathrm{C}$ ). Aparentemente, a tripa-de-sapo foi a espécie vegetal que mais foi influenciada pelo decréscimo na temperatura do ar.

TABELA 2. Valores (média \pm desvio) de concentração efluente de DQO $\left(\mathrm{g} \mathrm{m}^{-3}\right)$ nos SACs tratando ARS filtrada. Values (average \pm deviation) of effluent concentration of COD $\left(\mathrm{g} \mathrm{m}^{-3}\right)$ in the CWs treating filtered swine wastewater.

\begin{tabular}{lcccc}
\hline Período & $\begin{array}{c}\text { SAC1 } \\
\text { taboa }\end{array}$ & $\begin{array}{c}\text { SAC2 } \\
\text { tripa-de-sapo }\end{array}$ & $\begin{array}{c}\text { SAC3 } \\
\text { tifton } 85\end{array}$ & $\begin{array}{c}\text { SAC4 } \\
\text { Misto }\end{array}$ \\
\hline Verão & $122 \pm 62$ & $94 \pm 78$ & $121 \pm 90$ & $128 \pm 125$ \\
Outono/Inverno & $155 \pm 42$ & $207 \pm 118$ & $140 \pm 57$ & $130 \pm 38$ \\
\hline
\end{tabular}

SONG et al. (2006), pesquisando SACs tratando esgotos domésticos na China, também observaram que as concentrações de DQO efluentes eram maiores no período de outono/inverno. No entanto, tais pesquisas foram realizadas em SACs de escoamento livre (superficial).

Nas Tabelas 3 e 4, estão apresentados, respectivamente, os valores de concentração relativa $\left(\mathrm{C} / \mathrm{C}_{\mathrm{a}}\right)$ de DQO nas amostras coletadas em diferentes posições dentro dos quatro SACs, respectivamente, nos períodos de verão e outono/inverno. 
TABELA 3. Média dos valores de relação adimensional entre concentração de DQO residente e afluente $(\mathrm{C} / \mathrm{Ca})$ nos SACs cultivados com diferentes espécies vegetais, no período de verão. Average values of the dimensionless ratio among resident and affluent COD concentration $(\mathrm{C} / \mathrm{Ca})$ in the $\mathrm{CWs}$ cultivated with different species, in the summer period.

\begin{tabular}{ccccc}
\hline $\begin{array}{c}\text { TRH } \\
(\mathrm{d})\end{array}$ & $\begin{array}{c}\text { SAC1 } \\
\text { taboa }\end{array}$ & $\begin{array}{c}\text { SAC2 } \\
\text { tripa-de-sapo }\end{array}$ & $\begin{array}{c}\text { SAC3 } \\
\text { tifton } 85\end{array}$ & $\begin{array}{c}\text { SAC4 } \\
\text { misto }\end{array}$ \\
\hline 0,0 & 1,000 & 1,000 & 1,000 & 1,000 \\
1,1 & 0,213 & 0,429 & 0,233 & 0,221 \\
2,1 & 0,229 & 0,130 & 0,158 & 0,163 \\
3,2 & 0,155 & 0,224 & 0,184 & 0,235 \\
4,3 & 0,142 & 0,145 & 0,156 & 0,154 \\
5,3 & 0,292 & 0,155 & 0,061 & 0,133 \\
6,4 & 0,127 & 0,064 & 0,067 & 0,076 \\
\hline
\end{tabular}

TABELA 4. Média dos valores de relação entre concentração de DQO residente e afluente (C/Ca) nos SACs cultivados com diferentes espécies vegetais, no período de outono/inverno. Average values of the dimensionless ratio among resident and affluent COD concentration $(\mathrm{C} / \mathrm{Ca})$ in the $\mathrm{CWs}$ cultivated with different species, in the autumn/winter period.

\begin{tabular}{ccccc}
\hline $\begin{array}{c}\text { TRH } \\
(\mathrm{d})\end{array}$ & $\begin{array}{c}\text { SAC1 } \\
\text { taboa }\end{array}$ & $\begin{array}{c}\text { SAC2 } \\
\text { tripa-de-sapo }\end{array}$ & $\begin{array}{c}\text { SAC3 } \\
\text { tifton } 85\end{array}$ & $\begin{array}{c}\text { SAC4 } \\
\text { Misto }\end{array}$ \\
\hline 0,0 & 1,000 & 1,000 & 1,000 & 1,000 \\
1,1 & 0,416 & 0,374 & 0,500 & 0,378 \\
2,1 & 0,302 & 0,209 & 0,197 & 0,238 \\
3,2 & 0,237 & 0,170 & 0,168 & 0,144 \\
4,3 & 0,182 & 0,178 & 0,158 & 0,115 \\
5,3 & 0,289 & 0,219 & 0,144 & 0,132 \\
6,4 & 0,121 & 0,151 & 0,113 & 0,076 \\
\hline
\end{tabular}

Alguns dados foram perdidos, principalmente nos obtidos de amostras coletadas no início dos SACs, em função da dificuldade de retirada de amostras sem captação de parte do material orgânico em suspensão, próximo ao ponto de sucção da água residuária. Isso fez com que se obtivessem resultados que não refletiam a realidade do sistema. Essa interferência nos resultados deu-se nas coletas efetuadas no período de outono/inverno (Tabela 4), ocasião em que os interstícios do material poroso se encontravam mais preenchidos com material orgânico, já que os SACs já haviam recebido 3 meses de aplicação contínua de água residuária da suinocultura.

Os parâmetros ajustados para a utilização do modelo matemático de cinética de primeira ordem modificado por BRASIL et al. (2007), obtidos para dados coletados nos SACs, quando cultivados nos períodos de verão e outono/inverno, estão apresentados na Tabela 5. Como houve diferença significativa $(\mathrm{p}<0,15)$ entre os valores de $\mathrm{k}_{\mathrm{v}}$ ' para os períodos, os valores obtidos foram mantidos em dois grupos separados. Tendo em vista que não foi encontrada diferença significativa entre os valores de $\mathrm{k}_{\mathrm{v}}$ ' ajustados para cada SAC, foi apresentada uma equação que expressasse o comportamento geral dos SACs no período de verão e outra para o período de outono/inverno.

Verifica-se, avaliando-se as curvas de $\mathrm{C} / \mathrm{C}_{\mathrm{a}}$ como função do tempo de retenção hidráulica, que o comportamento foi semelhante entre os SACs, para ambos os períodos, com exceção feita ao sistema cultivado com a tripa-de-sapo. Os SACs (exceção feita ao SAC 2) apresentaram coeficientes de remoção $\left(\mathrm{k}_{\mathrm{v}}{ }^{\prime}\right)$ mais elevados no período de verão. $\mathrm{Na}$ interpretação dos valores dos parâmetros ajustados, é preciso levar em consideração que, quanto maior o valor de $\mathrm{k}_{\mathrm{v}}{ }^{\prime}$, maior $\mathrm{o}$ decréscimo na relação $C / C_{a}$ no início dos SACs. Em relação ao parâmetro $n$, quanto maior o seu valor, maior a taxa de remoção de DQO, notadamente a partir dos primeiros $4 \mathrm{~m}$ do início dos SACs. 
TABELA 5. Valores dos parâmetros do modelo de cinética de primeira ordem modificado por BRASIL et al. (2007), ajustados aos dados de concentração relativa de DQO (adimensional), como função do TRH (d), nos SACs cultivados com diferentes espécies vegetais. Parameters values of the first order kinetic model modified by BRASIL et al. (2007), adjusted to data of relative concentration of COD (dimensionless), as function of HRT (d), in the CWs cultivated with different species.

\begin{tabular}{|c|c|c|c|c|c|c|}
\hline \multirow{2}{*}{ SACs } & \multicolumn{3}{|c|}{ Verão } & \multicolumn{3}{|c|}{ Outono/Inverno } \\
\hline & $\mathrm{k}_{\mathrm{v}}{ }^{\prime}\left(\mathrm{d}^{-1}\right)$ & $\mathrm{n}$ & $\mathrm{R}^{2}$ & $\mathrm{k}_{\mathrm{v}}{ }^{\prime}\left(\mathrm{d}^{-1}\right)$ & $\mathrm{n}$ & $\mathrm{R}^{2}$ \\
\hline Taboa & 1,52 & 0,07 & 0,961 & 0,88 & 0,38 & 0,968 \\
\hline Tripa-de-sapo & 0,94 & 0,52 & 0,960 & 1,07 & 0,32 & 0,981 \\
\hline Tifton 85 & 1,39 & 0,30 & 0,989 & 0,78 & 0,63 & 0,973 \\
\hline Misto & 1,44 & 0,20 & 0,984 & 0,96 & 0,53 & 0,996 \\
\hline
\end{tabular}

Observando-se os valores obtidos após o ajuste do modelo aos dados coletados no período de verão (Tabela 5), verifica-se que, embora a taboa tenha proporcionado maior remoção da DQO, nos primeiros $4 \mathrm{~m}$ do SAC ( $\mathrm{k}_{\mathrm{v}}$ ' igual a $\left.1,52 \mathrm{~d}^{-1}\right)$, também foi ela que proporcionou a menor remoção a partir deste ponto (n igual a 0,07). Tais observações também foram feitas por BRASIL et al. (2007), que, estudando SACs cultivados com taboa, verificaram que a maior parte da remoção de material orgânico ocorreu nos 4 metros iniciais dos SACs. Tal fato deve-se, principalmente, ao efeito filtrante do meio suporte e sistema radicular das plantas.

No que se refere aos parâmetros ajustados a partir de dados coletados no inverno, verificou-se que a tripa-de-sapo apresentou maior remoção de DQO nos primeiros $4 \mathrm{~m}$ do SAC, seguida de menor remoção a partir desse ponto, tendo sido ajustados para este SAC o maior valor de $\mathrm{k}_{\mathrm{v}}$ ' $\left(1,07 \mathrm{~d}^{-1}\right)$ e o menor valor de $\mathrm{n}(0,32)$, dentre todos os sistemas avaliados. No período mais frio, os valores de $\mathrm{k}_{\mathrm{v}}$ ' ajustados para as equações obtidas para os SACs 1, 2 e 4 ficaram entre 0,78 e $0,96 \mathrm{~d}^{-1}$, e os de $\mathrm{n}$, entre 0,38 e 0,63 .

Como ressaltado anteriormente, não foram observadas diferenças significativas $(p>0,15)$ entre os SACs dentro de cada período. Dessa maneira, são apresentadas, nas Figuras 2 e 3, as curvas que expressam o comportamento geral dos SACs no período de verão e outono/inverno. As equações matemáticas contidas nos gráficos foram obtidas pela regressão dos valores médios das concentrações residentes em cada período.

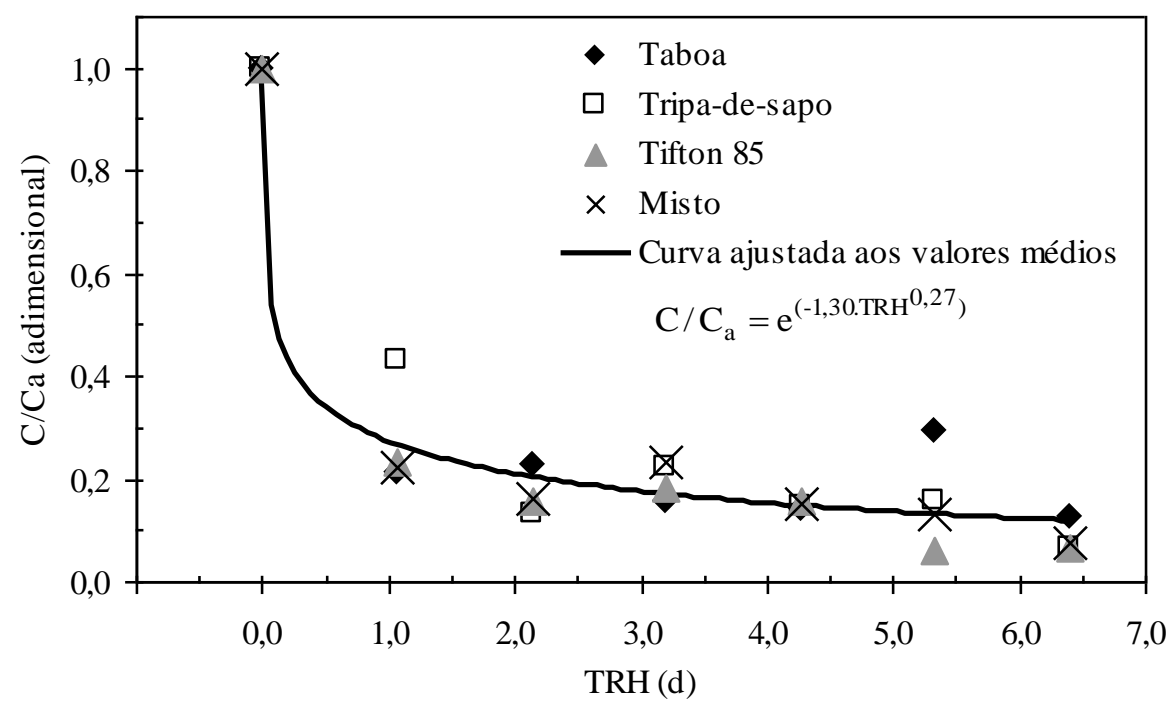

FIGURA 2. Concentração relativa de DQO na água residuária em tratamento nos SACs cultivados com diferentes espécies vegetais, no período de verão. Relative concentration of COD in wastewater in CWs cultivated with different species, in summer period. 


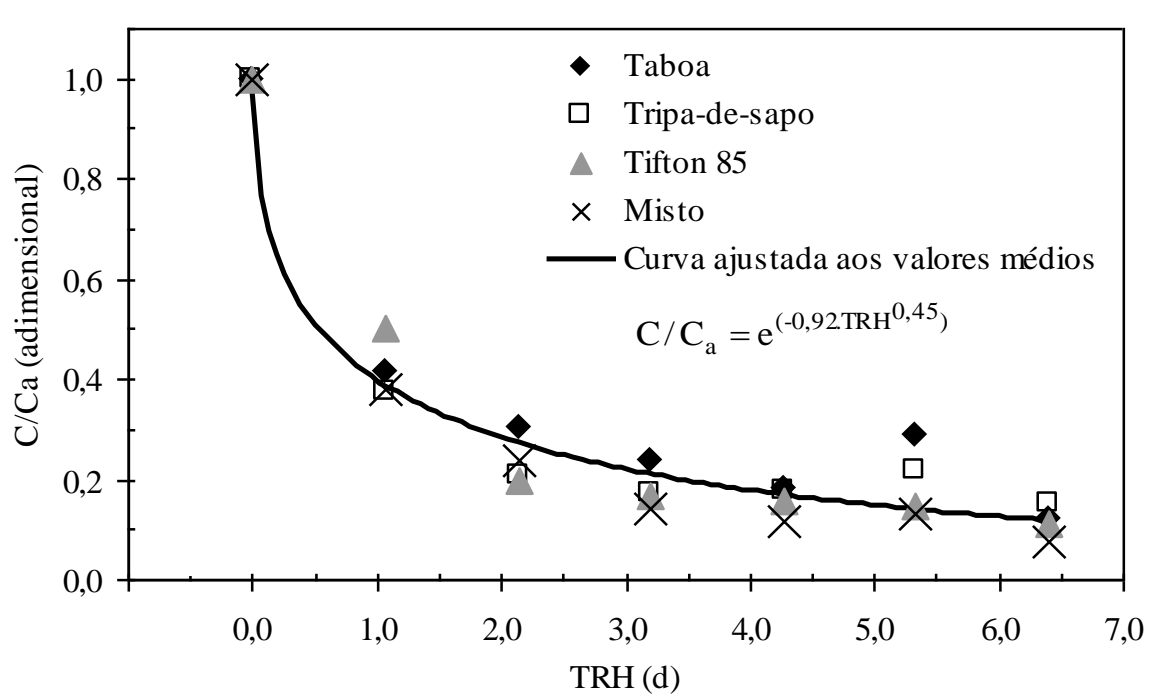

FIGURA 3. Concentração relativa de DQO na água residuária em tratamento nos SACs cultivados com diferentes espécies vegetais, no período de outono/inverno. Relative concentration of COD in wastewater in the CWs cultivated with different species, in the autumn/winter period.

As equações apresentadas nas Figuras 2 e 3 descreveram de forma adequada o decaimento de matéria orgânica nos SACs. Os valores de $\mathrm{R}^{2}$ foram de 0,963 (período de verão) e 0,970 (outono/inverno). Tais resultados indicam a aplicabilidade do modelo proposto por BRASIL et al. (2007), mesmo para efluentes não domésticos.

Quando ponderados os dois períodos, observou-se que os parâmetros obtidos para o SAC 4 foram os que mais se aproximaram dos parâmetros médios. Tal fato é justificado pela presença das três espécies nesse sistema, fazendo com que ele apresentasse, naturalmente, uma configuração "média". De fato, o objetivo da implantação de SACs multivegetados (mistos) é a otimização das habilidades e minimização das limitações apresentadas por cada planta.

Aparentemente, a remoção de DQO no verão é mais pronunciada no início do percurso dos SACs (valor de $\mathrm{k}_{\mathrm{v}}$ ' de $1,30 \mathrm{~d}^{-1}$ versus $0,92 \mathrm{~d}^{-1}$ para o outono/inverno). Consequentemente, a resistência à degradação, medida pelo inverso do parâmetro $\mathrm{n}$, também é maior nesse período, uma vez que a resistência à decomposição da matéria orgânica tende a aumentar após a oxidação do material de caráter mais lábil.

O coeficiente de remoção ou decaimento da matéria orgânica depende das características da própria matéria orgânica, temperatura do líquido e da presença ou não de substâncias inibidoras (SPERLING, 2005). BRASIL et al. (2007) encontraram em SACs cultivados com taboa, utilizados no tratamento de esgoto doméstico primário, ajuste do valor de $1,62 \mathrm{~d}^{-1}$ para o parâmetro $\mathrm{k}_{\mathrm{v}}$ ' e de 0,25 para n, valores relativamente próximos aos encontrados neste trabalho, no período de verão. Os mesmos autores ajustaram a equação de cinética de $1^{\text {a }}$ ordem modificada aos dados obtidos por VALENTIM (2003), em SAC cultivado com taboa, utilizado no tratamento de esgoto doméstico primário, e obtiveram valores de $\mathrm{k}_{\mathrm{v}}$ ' igual a $0,83 \mathrm{~d}^{-1}$ e os de $\mathrm{n}$ de 0,36 , mais próximos aos obtidos no outono/inverno, neste experimento. Possivelmente, os resultados obtidos por VALENTIM (2003) tenham sido gerados sob condições climáticas semelhantes às dominantes no período de outono/inverno de operação dos SACs deste trabalho.

\section{CONCLUSÕES}

A maior parte da remoção de DQO da água residuária em tratamento ocorreu nos primeiros $4 \mathrm{~m}$ dos SACs. 
A taxa de remoção de carga orgânica não foi influenciada pela espécie vegetal cultivada; entretanto foi influenciada pelo período do ano de operação dos SACs.

No período de verão (temperatura média do ar entre 19,0 e $25,0{ }^{\circ} \mathrm{C}$ ), os valores obtidos para $\mathrm{k}_{\mathrm{v}}$ ' foram de $1,30 \pm 0,10 \mathrm{~d}^{-1}$; e os de $\mathrm{n}$, iguais a $0,27 \pm 0,06$; já no período de outono/inverno (temperatura média do ar entre 10,8 e $21,9{ }^{\circ} \mathrm{C}$ ), os valores de $\mathrm{k}_{\mathrm{v}}$ ' foram menores $\left(0,92 \pm 0,06 \mathrm{~d}^{-1}\right)$, e os de $n$, maiores $(0,45 \pm 0,05)$.

\section{AGRADECIMENTOS}

Ao Conselho Nacional de Desenvolvimento Científico e Tecnológico (CNPq), pelo financiamento do projeto.

\section{REFERÊNCIAS}

APHA/AWWA/WEF. Standard methods for the examination of water and wastewater. $21^{\text {th }}$ ed. Washington: APHA, 2005. 1268 p.

BORGES, A.C.; MATOS, A.T.; CALIJURI, M.C.; OLIVEIRA, G.H.H.; ROLDÃO, J.S.F. Uso de traçadores para avaliação da hidrodinâmica de sistemas alagados construídos operando sob condições climáticas tropicais. Engenharia Agrícola, Jaboticabal, v.29, n.2, p.301-310, 2009.

BRASIL, M.S.; MATOS, A.T.; SILVA, C.M.; CECON, P.R.; SOARES, A.A. Modeling of pollution removal $\mathrm{n}$ constructed wetlands with horizontal subsurface flow. Journal of Agricultural Engineering Research, London, v.13, n.2, p.48-56, 2007.

COPAM/CERH. Deliberação Normativa Conjunta COPAM/CERH n ${ }^{\circ} .1$ de 5 de maio de 2008. Belo Horizonte, 2008.

FIA, R.; MATOS, A.T.; MATOS, M.P.; ABREU, E.C.; FIA, F.R.L. Tratamento das águas do processamento dos frutos do cafeeiro em filtro anaeróbio seguido por sistema alagado construído: I - remoção de matéria orgânica. Engenharia Agrícola, Jaboticabal, v.30, n.6, p.1.191-1.202, 2010.

HUNT, P.G.; POACH, M.E. State of the art for animal wastewater treatment in constructed wetlands. Water Science and Technology, Londres, v.44, n.11-12, p.19-25, 2001.

KADLEC, R.H.; WALLACE, S.D. Treatment Wetlands. Boca Raton: CRC Press, 2009. 1.016 p.

MAGALHÃES, M.A.; MATOS. A.T.; DENÍCULI, W.; TINOCO, I.F.F. Operação de filtros orgânicos no tratamento de águas residuárias de suinocultura. Revista Brasileira de Engenharia Agrícola e Ambiental, Campina Grande, v.10, n.2, p. 472-478, 2006.

MATOS, A.T.; ABRAHÃO, S.S.; BORGES, A.C.; MATOS, M.P. Influência da taxa de carga orgânica no desempenho de sistemas alagados construídos cultivados com forrageiras. Engenharia Sanitária e Ambiental, Rio de Janeiro, v.15, n.1, p.83-92, 2010 b.

MATOS, A.T.; ABRAHÃO, S.S.; LO MONACO, P. A.L.; SARMENTO, A.P.; MATOS, M.P. Capacidade extratora de plantas em sistemas alagados utilizados no tratamento de águas residuárias de laticínios. Revista Brasileira de Engenharia Agrícola e Ambiental, Campina Grande, v.14, n.12, p.1.311-1.317, 2010a.

MATOS, A.T.; ABRAHÃO, S.S.; PEREIRA, O.G. Desempenho agronômico de capim tifton 85 (Cynodon spp) cultivado em sistemas alagados construídos utilizados no tratamento de água residuária de laticínios. Revista Ambi-Água, Taubaté, v.3, n.1, p.43-53, 2008.

REED, S.C.; CRITES, R.W.; MIDDLEBROOKS, E.J. Natural systems for management and treatment, New York: McGraw-Hill, 1995. 435 p.

SONG, Z.; ZHENG, Z.; LI, J.; SUN, X.; HAN, X.; WANG, W.; XU, M. Seasonal and annual performance of a full-scale constructed wetland system for sewage treatment in China. Ecological Engineering, Amsterdam, v.26, n.3, p.272-282, 2006. 
SPERLING, M. VON. Introdução à qualidade das águas e ao tratamento de esgotos. Belo Horizonte: DESA/UFMG, 2005. 243p. (Princípios do tratamento biológico de águas residuárias, v.1)

TROTTER, E.A.; THONSON, B.; COLEMAN, R. Evaluation of a subsurface flow wetland processing sewage from the Sevilleta LTER field station. Las Cruces: New Mexico Water Resources Research Institute, 1994. 52 p. (WRRI Report, 287).

VALENTIM, M.A.A. Desempenho de leitos cultivados ("construted wetland") para tratamento de esgoto: contribuições para concepção e operação. Tese (Doutorado) - Faculdade de Engenharia Agrícola, Universidade Estadual de Campinas, Campinas, 2003. 210 p.

VYMAZAL, J.; KRÖPFELOVÁ, L. Removal of organics in constructed wetlands with horizontal sub-surface flow: A review of the field experience. Science of the Total Environment, Amsterdam, v.407, n.13, p.3.911-3.922, 2009. 